\title{
Acciones para una universidad saludable: Impacto sobre la salud mental y física de los jóvenes Actions for a healthy university: Impact on mental and physical health in young people
}

Gema Sanchis-Soler, Miguel García-Jaén, Sergio Sebastia-Amat, Cristian Diana-Sotos, Juan Tortosa-Martinez Universidad de Alicante (España)

Resumen. La inactividad física y sedentarismo se encuentran presentes en un alto porcentaje de los estudiantes universitarios, hecho que se ha visto acentuado tras la crisis sanitaria vivida recientemente a causa de la COVID-19. El ejercicio físico como parte de los programas de las Universidades Saludables se presenta como una posible estrategia para afrontar esta problemática. El objetivo de este estudio piloto fue analizar los efectos de un programa de entrenamiento sobre el nivel de actividad física (AF), salud mental y composición corporal en estudiantes universitarios sedentarios. Se diseñó un estudio longitudinal de medidas repetidas. 14 estudiantes fueron evaluados en tres momentos temporales: al iniciar el programa de entrenamiento, tras su finalización y dos semanas tras la finalización del programa. Los diferentes instrumentos utilizados fueron los siguientes: Cuestionario Internacional de Actividad Física (nivel de AF), cuestionario DASS-21 (nivel de estrés, depresión y ansiedad) y Cuestionario Pittsburgh Sleep Quality Index (calidad del sueño) cinta métrica corporal (perímetros) y bioimpedancia (peso, IMC, \% masa grasa y \% masa muscular). Tras cinco semanas de entrenamiento y dos semanas post-entrenamiento se observaron mejoras significativas en el nivel de AF, salud mental y calidad del sueño de los participantes $(p<, 05)$. La masa muscular fue la única variable relacionada con la composición corporal que mejoró significativamente tras cinco semanas de ejercicio físico supervisado $(p=, 04)$. Los resultados del presente estudio demostraron que las acciones de las universidades basadas en programas de entrenamiento saludables mejoran el nivel de AF, la salud mental y la composición corporal de los estudiantes universitarios.

Palabras clave. Entrenamiento domiciliario, salud mental, calidad del sueño, actividad física, universitarios.

Abstract. University students frequently show high levels of physical inactivity as well as a sedentary lifestyle. This fact has been increased after the recent health crisis caused by the COVID-19. Physical exercise, as part of the programs of the Healthy Universities, could be a strategy to face this problem. The purpose of this pilot study was to analyze the effects of an exercise program on the level of physical activity, mental health, and body composition in sedentary university students. A longitudinal study with repeated measures was designed. 14 students were evaluated at three different moments: at the beginning of the exercise program, after program completion, and two weeks after the end of the program. The followings instruments were used: International Physical Activity Questionnaire (level of physical activity), DASS-21 questionnaire (level of stress, depression, and anxiety), Pittsburgh Sleep Quality Index Questionnaire (quality of sleep), a body tape measure (perimeters), and a body composition monitor (weight, BMI, \% fat mass and \% muscle mass). After five weeks of intervention and two weeks postintervention, the levels of physical activity, mental health and quality of sleep were improved $(p<.05)$. Regarding body composition, muscle mass was the only variable that improved after five weeks of exercise program $(p=.04)$. The results of the present study showed that the actions of universities based on healthy training programs improve the level of physical activity, mental health, and body composition of university students.

Keywords. Home workout, mental health, sleep quality, physical activity, university.

\section{Introducción}

La virulencia del SARS-CoV2 obligó a implantar restricciones y medidas para intentar frenar su avance (toques de queda, cierres de comercios, limitaciones en las reuniones sociales y confinamiento domiciliario, entre otras) (Güner et al., 2020; Monge et al., 2021). Así pues, el confinamiento domiciliario supuso para la mayoría de la población cambios en las rutinas diarias y

Fecha recepción: 03-11-21. Fecha de aceptación: 01-02-22

Sergio Sebastia Amat

sergio.sebastia@ua.es estilos de vida, tales como modificaciones en los hábitos alimenticios o disminución del nivel de actividad física (Castañeda-Babarro et al., 2020; DeschasauxTanguy et al., 2021). De hecho, y a pesar de que con anterioridad a la pandemia la inactividad física ya era considerada un problema económico y de salud pública a nivel mundial (Kruk, 2014), el porcentaje de gente inactiva y sedentaria creció durante el periodo pandémico, siendo los estudiantes universitarios uno de los sectores más afectados (Castañeda-Babarro et al., 2020; Díaz et al., 2019; Gallè et al., 2020; Gallo et al., 2020).

A las consecuencias negativas para la salud deriva- 
das del estilo de vida sedentario e inactivo (obesidad, hipertensión, diabetes, colesterol, etc.) (Arocha Rodulfo, 2019), se sumaron la crisis económica, sanitaria y social (Ali \& Alharbi, 2020; Nicola et al., 2020), derivando todo ello en el aumento de problemas físicos, psicológicos y/o de salud mental (Mancini, 2020; Vindegaard \& Benros, 2020). En relación a estos últimos, destacamos por su alta prevalencia entre la población universitaria, la depresión, la ansiedad y el estrés (Maia \& Dias, 2020), así como una disminución en la calidad del sueño (Marelli et al., 2021). De hecho, Cao et al. (2020) tras analizar a 7143 estudiantes de medicina de la facultad de Changzhi, concluyeron que el 24,9\% de los estudiantes habían padecido o padecían ansiedad, de los cuales un $0,9 \%$ experimentó ansiedad severa y un $21,3 \%$ ansiedad leve. Asimismo, demostraron que estos niveles de ansiedad estaban directamente relacionados con el lugar de residencia, la fuente de ingresos de los padres y con el contagio por COVID-19 de un familiar o conocido. Del mismo modo, Padrón et al. (2021) con el objetivo de conocer el daño psicológico causado por el virus en los estudiantes españoles, analizaron a una muestra de 932 alumnos/as universitarios, observando que el 61,2\% y $65,8 \%$ de los participantes sufrían altos niveles de ansiedad y depresión, respectivamente, relacionando estos estados con la incertidumbre sobre el futuro académico, la sobrecarga de tareas, el empeoramiento de los conflictos interpersonales y las restricciones en el contacto social. Este factor de salud mental a su vez se relacionó con la calidad del sueño (Ghrouz et al., 2019), la cual se vio alterada y deteriorada durante el periodo de confinamiento domiciliario (Franceschini et al., 2020).

Ante esta problemática, son muchos los estudios que apoyan y corroboran los beneficios de la práctica de AF en la prevención y/o mejora de la salud en los jóvenes. Hace una década, autores como Olmedilla et al. (2010) relacionaron la práctica de ejercicio físico con menores niveles de ansiedad y depresión en universitarias. Más recientemente, Torales et al. (2018) hicieron hincapié en los beneficios del ejercicio físico sobre el estado de ánimo, calidad del sueño, estrés y capacidad cognitiva. Del mismo modo, son muchos los trabajos que destacan el papel que juega la AF en la mejora de la composición corporal, disminución de la resistencia a la insulina y colesterol, así como adherencia a la dieta mediterránea entre la población más joven (López-Nuevo et al., 2021; Sun et al., 2020).

Por todo ello, resulta necesario la promoción y desarrollo de programas para el fomento de la salud de los jóvenes (Monteiro et al., 2019). Se trata de educar y aportar a los ciudadanos las estrategias y conocimientos necesarios para que sean capaces de identificar comportamientos incorrectos y saber cómo corregirlos. Así pues, la Organización de las Naciones Unidas (ONU) incluye la salud y el bienestar como uno de los puntos para alcanzar el desarrollo sostenible, considerando que «garantizar una vida sana y promover el bienestar en todas las edades es esencial para el desarrollo sostenible» (Organización de las Naciones Unidas, 2015). Ejemplo de ello lo encontramos en la campaña puesta en marcha la tercera semana de septiembre de 2020 por la OMS y la Organización Paramericana de la Salud (OPS). Ambos organismos, bajo el lema «Juntos más Fuertes», insistieron en la necesidad e importancia de mantener y mejorar la salud mental de la población (Organización Mundial de la Salud \& Organización Paramericana de la Salud, 2020). De igual modo, dentro del marco de acción de la Red Española de Universidades Saludables (REUS), se aboga por la creación de espacios, ofertas de servicios y actividades, así como el fomento de la investigación para la promoción de la salud y aumento de la práctica de AF dentro de la comunidad universitaria (Ministerio de Sanidad, 2008). Este movimiento, junto con la iniciativa autonómica de la RedValenciana de Universidades Públicas Saludables (RVUPS), surgió para dar respuesta a la necesidad de reforzar el papel de las universidades como entidades promotoras de la salud y el bienestar de sus estudiantes, personal docente e investigador, personal de administración y servicios y, en última instancia, de la sociedad en su conjunto (Cervera Burriel et al., 2013). De esta manera, la universidad cumple con su propósito de liderar y apoyar los procesos de cambio social y contribuir al cumplimiento de los objetivos de desarrollo sostenible (Sachs, 2012).

Sin embargo, a pesar de los esfuerzos de estas iniciativas, se siguen detectado ciertas carencias. En este sentido, Martínez-Sanz et al. (2018) señalaron que, a pesar de que en la Comunidad Valenciana las universidades miembros de la RVUPS habían puesto en marcha diferentes actividades, cursos y talleres para la promoción de la AF, la salud y la calidad de vida, éstas quedaban en ocasiones alejadas de la oferta e iniciativas dirigidas por la Universidades Españolas miembro de la REUS. Por tanto, los autores incidieron en la necesidad de actuaciones más directas y eficaces en la promoción de la AF y salud con programas completos de ejercicio y formación, un seguimiento de los participantes y los programas llevados a cabo, así como un sistema de comunicación e información que garantice la correcta difusión (Cerrillo Borja et al., 2019; Martínez-Sanz et al., 2018). 
En este contexto, con el objetivo de promover hábitos saludables y de mejora de la salud entre los estudiantes universitarios, se requiere de acciones que garanticen el éxito de las intervenciones saludables promovidas por las universidades. Ante esta necesidad, el presente trabajo se presenta con el objetivo de analizar los efectos de un programa de entrenamiento sobre el nivel de AF, la salud mental y composición corporal de estudiantes universitarios sedentarios durante el curso 2020-2021. Por otro lado, se pretende determinar la validez de este tipo de programas para provocar la adherencia a la práctica de ejercicio físico de estudiantes universitarios.

\section{Metodología}

Se presenta un estudio piloto longitudinal de medidas repetidas, con una selección de la muestra no probabilística e intencional.

\section{Participantes}

16 estudiantes del grado en Maestro de Educación Primaria e Infantil de la Universidad de Alicante participaron en el programa piloto. Tras aplicar los criterios de inclusión y exclusión, la muestra final se compuso de 14 participantes, 10 mujeres y 4 hombres (edad media \pm DE: $20,86 \pm 1,79$ ). Según los criterios previamente establecidos, se incluyeron en el estudio estudiantes universitarios de entre 18 a 30 años, que no hubiesen realizado por cuenta propia actividad o participado en ningún programa de ejercicio físico en los últimos seis meses. Se excluyeron todos aquellos participantes que no completaron como mínimo el 80\% de las sesiones o no cumplían con alguno de los criterios de inclusión establecidos. Todos los participantes fueron informados de las características del estudio y firmaron un consentimiento informado para participar. Este estudio se realizó siguiendo las directrices de la Declaración de Helsinki (2013).

\section{Variables $e$ instrumentos de evaluación}

Por lo que respecta al nivel de AF, este se determinó mediante el Cuestionario Internacional de AF (IPAQ) versión corta, compuesto por nueve ítems y tres niveles de AF: bajo (AF<600 METs-min/semana); medio (AF $\geq 600-\leq 3000 \mathrm{METs}-\mathrm{min} /$ semana) y alto (AF>3.000 METs-min/semana) (Toloza \& GómezConesa, 2007).

El nivel de depresión, ansiedad y estrés se evaluó con el cuestionario DASS-21, compuesto por 21 ítems organizados en tres dimensiones (Depresión, Ansiedad y Estrés) con siete ítems cada una (Alves Apóstolo et al., 2006). La puntuación obtenida se interpretó siguiendo los criterios establecidos por el laboratorio de psicología clínica ClinikLab (ClinikLab, 2017) (Tabla 1).

En tercer lugar, la calidad del sueño se evaluó mediante el Cuestionario Pittsburgh Sleep Quality Index (PSQI), compuesto por 19 ítems (Lomelí et al., 2008). Con una puntuación máxima posible de 21 puntos, se consideró una peor calidad del sueño cuanto más alta era la puntuación obtenida (Buysse et al., 1989). Por último, la composición corporal y el Índice Cintura Cadera (ICC) se registraron mediante un monitor de composición corporal (Omron BF511) y una cinta métrica corporal (Cescorf), respectivamente.

\begin{tabular}{|c|c|c|c|}
\hline & Depresión & Ansiedad & Estrés \\
\hline Ítems & $3,5,10,13,16,17$ y 21 & $2,4,7,9,15,19$ y 20 & $1,6,8,11,12,14$ y 18 \\
\hline \multicolumn{4}{|l|}{ Valoración } \\
\hline No presenta & $0-5$ & $0-3$ & $0-7$ \\
\hline Leve & $5-6$ & 4 & $8-9$ \\
\hline Moderado & 7-10 & $5-7$ & $10-12$ \\
\hline Severo & $11-13$ & $8-9$ & $13-16$ \\
\hline Muy severo & $\geq 14$ & $\geq 10$ & $\geq 17$ \\
\hline
\end{tabular}

\section{Procedimiento e intervención}

A lo largo de la intervención se realizaron tres evaluaciones: pre-intervención (Pre_int), post-intervención (Post_int) y dos semanas post-intervención (2Post_int) (Tabla 2). La difusión del programa se realizó mediante un anuncio por el Consejo de estudiantes de la Universidad de Alicante y por diferentes plataformas y redes sociales. Por otro lado, para poder mantener una comunicación diaria con los participantes se utilizó la aplicación «Telegram», mediante la cual se enviaba diariamente la sesión de entrenamiento junto a un video explicativo. Además, a través de esta aplicación los participantes compartieron sus retos y éxitos alcanzados.

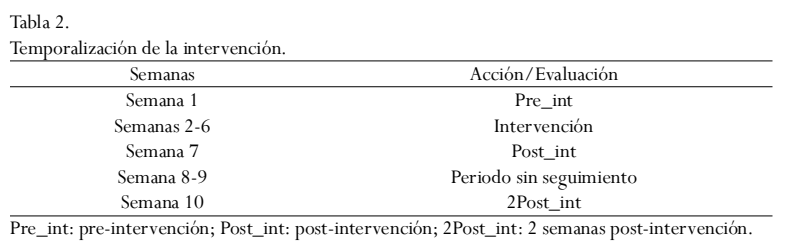

En el programa de ejercicio físico llevado a cabo, se combinaron sesiones de entrenamiento de acondicionamiento muscular (en el domicilio) con sesiones de entrenamiento aeróbico (caminatas). Para las sesiones de acondicionamiento muscular se estableció una progresión en frecuencia, volumen e intensidad. Así, durante las dos primeras semanas se realizaron dos sesiones de entrenamiento a la semana, aumentando a tres sesiones/semana para el resto del programa. Todas las sesio- 
nes se dividieron en dos bloques de ejercicios, para cada uno de los cuales se establecieron unas repeticiones y series determinadas. El número de series fue fijo para las dos semanas iniciales del programa, mientras que, para las tres semanas restantes se indicó a los participantes un tiempo en el cual debían realizar todas las series posibles respetando las repeticiones y tiempos de recuperación fijados por ejercicio y/o bloques (Boyle, 2017). En segundo lugar, por lo que respecta al entrenamiento aeróbico, con el objetivo de alcanzar un mayor compromiso y motivación por parte de los participantes, se les pidió que registraran el número de pasos diarios en el grupo de Telegram, adjuntando capturas de pantalla o imágenes de sus dispositivos. Además, se les recomendó que la caminata o paseo en bicicleta prevista para la sesión del domingo la realizaran por algún paraje natural o zona verde (Tabla 3).

El uso principal de la aplicación Telegram fue la supervisión del programa de ejercicio físico por parte de un graduado en Ciencias de la Actividad Física y el Deporte. Del mismo modo, mediante el uso de dicha aplicación se intentó crear el sentimiento de pertenencia a un grupo, compartiendo fotos y vídeos de sus entrenamientos, al mismo tiempo que recibían mensajes de motivación por parte del resto de los integrantes.

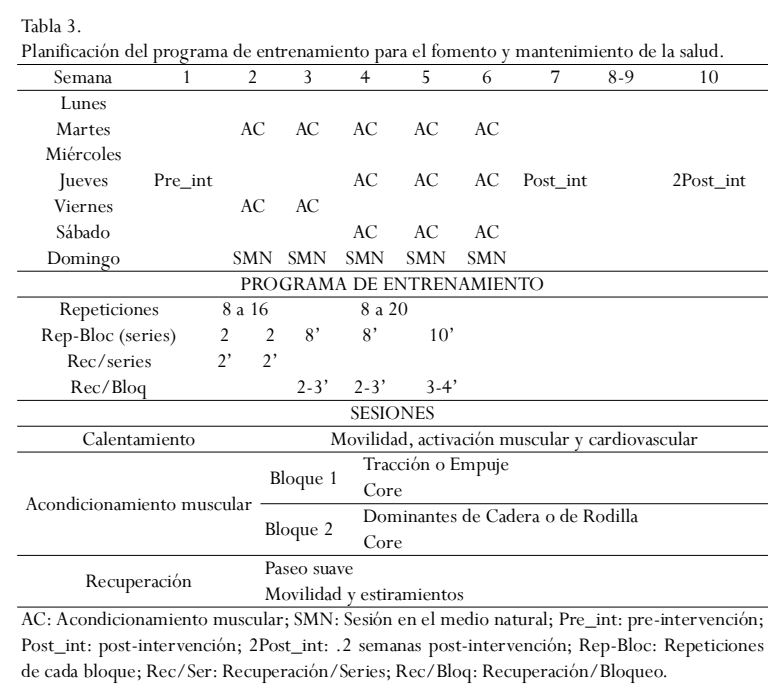

\section{Análisis de datos}

El procesamiento de los datos se realizó con el programa Statistical Package for Social Science v. 25 (IBM, Armonk, NY, USA). Se comprobó la normalidad de la muestra a través de la prueba de Shapiro-Wilk. En el caso del nivel de AF (IPAQ) se realizó la prueba ANOVA de medidas repetidas mientras que las variables que no cumplieron los criterios de normalidad (depresión, ansiedad, estrés, PSQI) se analizaron mediante la prueba de Friedman para muestras relacionadas. Cuando la prueba de Friedman mostró significancia, se realizó la prueba de contraste no paramétrica de Wilcoxon. Por lo que respecta a las variables relativas a la composición corporal sólo se pudo realizar el registro tras la intervención de cinco semanas. Así pues, se aplicó la prueba t de Student para muestras relacionadas en aquellas variables que seguían la normal (ICC, \% masa grasa y \% masa muscular), mientras que para el IMC y el peso se realizó la prueba de Wilcoxon. Se consideraron diferencias significativas aquellas con un valor de $p<, 05$. El tamaño del efecto (TE) se calculó utilizando la $(g)$ de Hedge para muestras pequeñas. El TE se definió como $<0,2$ trivial, 0,2-0,6 pequeño, 0,6-1,2 moderado y $>$ 1,2 grande (Lakens, 2013).

\section{Resultados}

En el presente estudio participaron 14 estudiantes universitarios de edades comprendidas entre los 18 y 30 años. El nivel AF reportado inicialmente fue medio y el número de horas que los participantes permanecieron sentados fue superior a ocho horas. Por lo que respecta a la composición corporal, el porcentaje de masa grasa fue relativamente alto para la franja de edad de los participantes. En la tabla 4 se presentan los descriptivos de la muestra.

\begin{tabular}{|c|c|}
\hline Variables & $\mathrm{M} \pm \mathrm{DE}$ \\
\hline Edad (años) & $20,86 \pm 1,79$ \\
\hline Peso (kg) & $68,78 \pm 25,53$ \\
\hline $\operatorname{IMC}(\mathrm{kg} / \mathrm{m} 2)$ & $23,39 \pm 4,71$ \\
\hline Masa Grasa (\%) & $31,74 \pm 7,40$ \\
\hline Masa Muscular (\%) & $28,33 \pm 4,99$ \\
\hline ICC & $0,76 \pm 0,05$ \\
\hline Tiempo sentado (horas) & $8,21 \pm 2,12$ \\
\hline AF (METs-min/semana) & $1721,56 \pm 925,71$ \\
\hline
\end{tabular}

Los valores iniciales del DASS-21 y PSQI, indicaron que los participantes presentaban indicios de depresión, estrés y ansiedad moderada, así como un leve problema en la calidad de sueño. El programa de entrenamiento llevado a cabo permitió mejorar todas estas variables en diferentes fases de la intervención (Tabla 5). Así pues, la depresión, la ansiedad y la calidad del sueño mejoraron de forma significativa tras cinco semanas de entrenamiento $(p=, 017 ; p=, 008 ; p=, 020$, respectivamente). No obstante, en el caso del estrés las mejoras no fueron significativas $(p=, 102)$. Tras dos semanas post intervención, tanto el nivel de depresión, estrés, ansiedad y calidad del sueño mejoraron de forma significativa respecto a los valores pre-intervención $(p=$ $, 004, p=, 001, p=, 007, p=, 009$, respectivamente). Por lo que respecta al nivel de AF, se observó un au- 
mento significativo tras las cinco semanas de entrenamiento $(p=, 001)$, así como tras dos semanas post intervención $(p=, 004)$. Por último, en cuanto a la composición corporal, únicamente se encontraron diferencias significativas en el porcentaje de masa muscular tras las 5 semanas de entrenamiento $(p=, 044)$.

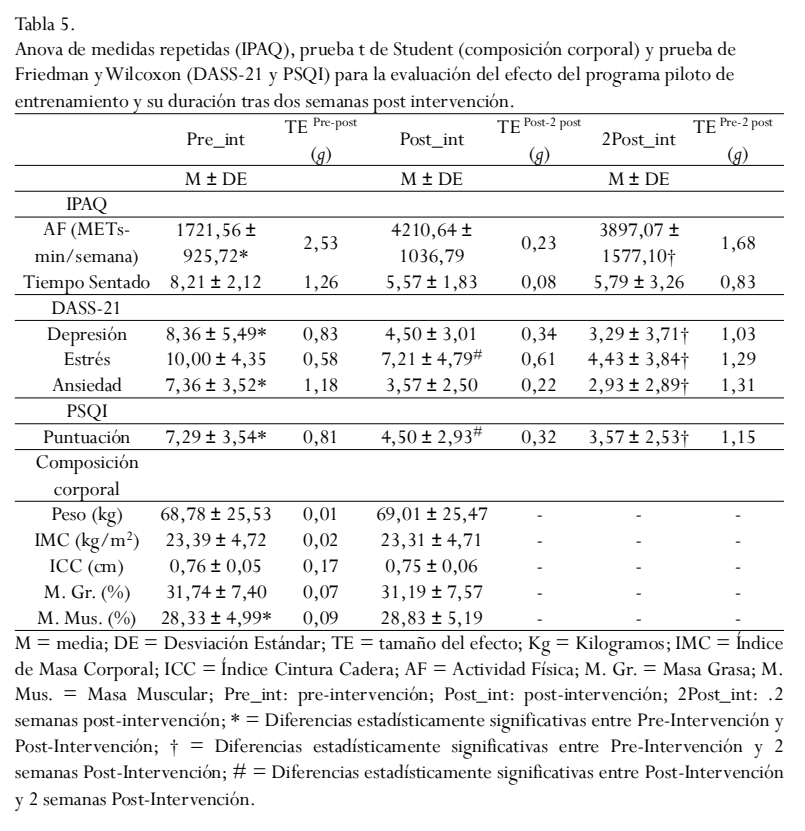

\section{Discusión}

El presente estudio tuvo como objetivos determinar los efectos de un programa de entrenamiento físico sobre la salud mental, calidad del sueño, composición corporal y nivel de AF en estudiantes universitarios sedentarios durante el curso académico 2020-2021, en el que la docencia se impartió siguiendo una metodología dual. Además, se observó la adherencia a corto plazo a la práctica de ejercicio físico por parte de los participantes.

En relación al nivel de AF, los participantes de nuestro estudio inicialmente mostraron un nivel de AF medio. Tal y como se ha indicado anteriormente, el estilo de vida inactivo y sedentario era un problema ya presente entre los estudiantes universitarios en los años previos a la pandemia, situación que se ha visto agravada por la crisis sanitaria actual (Gallo et al., 2020). Sin embargo, dejando a un lado las restricciones de movilidad y contacto social, estos comportamientos posiblemente se deban, entre otros, a la falta de tiempo, motivación, voluntad, habilidad, autoeficacia para la actividad física y cansancio diario provocado por las tareas académicas (Leyton-Román et al., 2021; Mella et al., 2021). Por ello, son necesarias intervenciones dirigidas hacia la motivación y aprendizaje de estrategias y hábi- tos de vida saludables de los participantes, poniendo a su disposición una planificación que les permita compatibilizar la práctica de ejercicio con los estudios universitarios. En este sentido, nuestra intervención permitió tras cinco semanas de entrenamiento que los participantes alcanzaran niveles de AF altos. Estos niveles se mantuvieron tras dos semanas post entrenamiento, superando en ambos casos a los resultados obtenidos por estudios previos (Boguszewski et al., 2021; Fayyad \& Dopsaj, 2021; Gallè et al., 2020). Los resultados obtenidos en el IPAQ tras la evaluación realizada tras dos semanas post intervención, podrían indicar la efectividad de este tipo de intervenciones sobre la adherencia a corto plazo a la práctica de ejercicio por parte de estudiante universitarios sedentarios. Inicialmente el 71,63\% de los participantes presentaban algún signo de estrés, el 64,3\% de depresión y el 78,57\% de ansiedad. Sin embargo, al finalizar el periodo de entrenamiento y a las dos semanas post intervención se observó una disminución de los estados de depresión, ansiedad y estrés. Posiblemente estas mejoras estén promovidas, entre otras, por el efecto fisiológico del ejercicio físico como consecuencia del aumento de endorfinas, ocasionando una disminución de la sensación de ansiedad y una mejora del autoconcepto (Mikkelsen et al., 2017). Estos resultados se muestran en línea con los obtenidos por Boguszewski et al. (2021) quienes coinciden en que uno de los principales motivos por los que los estudiantes realizan ejercicio físico es el mantenimiento de su salud mental.

En segundo lugar, la puntuación inicial obtenida en la calidad del sueño indicó que los participantes tenían dificultades leves para dormir. Tras la intervención, los participantes mostraron una mejor calidad de sueño. Además, manifestaron que les resultaba más fácil conciliar el sueño y que se despertaban con la sensación de tener más energía. Esto puede deberse al aumento de gasto energético ocasionado por el ejercicio físico, Coincidiendo así, con la investigación de Chang et al. (2016) en la que se observó que los estudiantes universitarios físicamente activos tenían mejor calidad del sueño. Por último, en relación a la composición corporal, los resultados del presente estudio muestran similitudes con el estudio de Cigerci \& Genc (2020), en el que se observó que la realización de 1 a 3 sesiones de entrenamiento de fuerza por semana es suficiente para obtener mejoras en el porcentaje de masa muscular.

Por tanto, y siguiendo con las campañas iniciadas por la OMS, así como por las acciones y propuestas desarrolladas por la REUS y RVUPS, los principios y carac- 
terísticas de la intervención presentada en este estudio, podrían solucionar algunas de las problemáticas detectadas en programas o campañas anteriores: falta de seguimiento de los programas y participantes, errores de promoción, horarios incompatibles y ausencia de tiempo y motivación por parte de los participantes (Cerrillo Borja et al., 2019; Martínez-Sanz et al., 2018; Santamaría et al., 2019). De esta forma, los autores sugieren que la presente propuesta debería tenerse en cuenta en el camino hacia la consecución de los objetivos de las universidades públicas saludables. Futuras investigaciones deberían incluir ofertas o programas de actividades con una promoción adecuada que permita su difusión entre todos los miembros de la comunidad universitaria. Por otro lado, se evidencia la necesidad de incluir estrategias de formación y motivación hacia el mantenimiento de hábitos de vida saludables, con un seguimiento periódico de los participantes. Aplicaciones de mensajería instantánea y/o canales informativos, como es el caso de la aplicación Telegram utilizada en el presente estudio, podría ser una herramienta útil y válida para alcanzar tales fines. Por último, es imprescindible adaptar las sesiones de entrenamiento a la disponibilidad horaria y temporal de cada sujeto. En este sentido, programar el entrenamiento de forma que pueda ser ejecutado tanto en una instalación deportiva como en el domicilio permite eliminar el tiempo dedicado al desplazamiento e invertirlo en la sesión de entrenamiento. Esto puedo suponer una ventaja para los/as estudiantes con altas cargas de trabajo intra y extra universitario, siendo a su vez una buena alternativa a la situación sanitaria actual, la cual ha provocado en numerosas ocasiones restricciones de aforos o cierres de centros deportivos.

El presente trabajo presenta algunas limitaciones. En primer lugar, se trata de un estudio piloto con una muestra reducida de estudiantes y sin grupo de control, por lo tanto, los resultados deben interpretarse con cautela, en base a estas consideraciones.

En este estudio únicamente se pudo realizar un seguimiento de dos semanas post-intervención, por lo que los resultados en cuanto a la adherencia deben valorarse únicamente a corto plazo. Con el objetivo de poder comprobar si el programa de entrenamiento tiene una repercusión sobre la adherencia a la práctica deportiva a medio y largo plazo, así como sobre el mantenimiento de la salud mental y calidad de sueño de los estudiantes, se requieren de futuros estudios que realicen un periodo de seguimiento mayor. En segundo lugar, a pesar de estandarizar el programa y controlar las cargas de en- trenamiento, la puesta en práctica del programa de actividad física en el domicilio de cada participante no fue estándar. Esta variabilidad en la puesta en práctica del programa por parte de los participantes se ha de considerar a la hora de valorar los efectos mostrados en este estudio.

Finalmente, las variables relacionadas con la composición corporal no pudieron medirse tras dos semanas post intervención debido al aumento de las restricciones ocasionadas por la COVID-19, por lo que no se dispone de esta información para valorar los efectos del programa en la composición corporal tras dos semanas de la acción.

\section{Conclusiones}

Los resultados del presente estudio muestran que un programa de ejercicio físico realizado en el marco de acciones saludables dentro de la universidad, puede ser una opción adecuada para la mejora y mantenimiento de los niveles de AF, de la salud mental y calidad del sueño en jóvenes universitarios. Además, emplear un seguimiento diario, implicar a los participantes en el proceso, aportar alternativas para el entrenamiento de fuerza en el propio domicilio y adaptar las sesiones a la disponibilidad horaria de los participantes, parece ser opciones válidas para promover la adherencia hacia la práctica de ejercicio físico en la comunidad universitaria.

\section{Referencias}

Ali, I., \& Alharbi, O. M. (2020). COVID-19: Disease, management, treatment, and social impact. Science of the Total Environment, 728(2020), 138861. https: / / doi.org/10.1016/j.scitotenv.2020.138861

Alves Apóstolo, J. L., Cruz Mendes, A., \& Aguiar Azeredo, Z. (2006). Adaptação para a língua portuguesa da depression, anxiety and stress scale (DASS). Rev Lat Am Enfermagem, 14(6).

Arocha Rodulfo, J. I. (2019). Sedentarism, a disease from xxi century. Clin InvestigArterioscler, 31(5), 233-240.https:/ /doi.org/10.1016/j.artere.2019.04.001

Boguszewski, D., Ochala, A., Adamczyk, J., Jasiñski, P., Szymañska, A., Obszyñska-Litwiniec, A., \& BiaBoszewski, D. (2021). Physical activity of physiotherapy students at the medical university of warsaw. Health Probl. Civiliz., 14(1). https://doi.org/ 10.5114/hpc. 2020.97678

Boyle, M. (2017). El entrenamiento funcional aplicado a los de- 
portes. Ediciones Tutor, SA.

Buysse, D. J., Reynolds III, C. F., Monk, T. H., Berman, S. R., \& Kupfer, D. J. (1989). The Pittsburgh Sleep Quality Index: a new instrument for psychiatric practice and research. Psychiatry research, 28(2), 193-213.

Cao, W., Fang, Z., Hou, G., Han, M., Xu, X., Dong, J., \& Zheng, J. (2020). The psychological impact of the COVID-19 epidemic on college students in China. Psychiatry Res., 287, 112934. https: / /doi.org/10.1016/ j.psychres.2020.112934

Castañeda-Babarro, A., Arbillaga-Etxarri, A., GutiérrezSantamaría, B., \& Coca, A. (2020). Physical activity change during COVID-19 confinement. Int J Environ Health Res, 17(18), 6878. https://doi.org/10.3390/ ijerph17186878

Cerrillo Borja, M., Martínez-Riera, J. R., \& López-Gómez, J. (2019). El movimiento de universidades saludables en España. RIdEC.

Cervera Burriel, F., Serrano Urrea, R., Vico García, C., Milla Tobarra, M., \& García Meseguer, M. J. (2013). Hábitos alimentarios y evaluación nutricional en una población universitaria. Nutr Hosp, 28(2), 438-446. https:/ /doi.org/10.3305/nh.2013.28.2.6303

Chang, S.-P., Shih, K.-S., Chi, C.-P., Chang, C.-M., Hwang, K.-L., \& Chen, Y.-H. (2016). Association between exercise participation and quality of sleep and life among university students in Taiwan. Asia Pac J Public Health, 28(4), 356-367. https://doi.org/10.1177/ 1010539516645160

Cigerci, A. E., \& Genc, H. (2020). The effect of strength training with different frequency on untrained university students. Phys. educ. stud, 24(4), 186-193. https: / / doi.org/ $10.15561 / 20755279.2020 .0401$

ClinikLab. (2017). DASS-21 . Recuperado octubre, 10, 2021, de https:/ / cliniklab.konradlorenz.edu.co/2020/09/ sintomas.html

Deschasaux-Tanguy, M., Druesne-Pecollo, N., Esseddik, Y., de Edelenyi, F. S., Allès, B., Andreeva, V. A., Baudry, J., Charreire, H., Deschamps,V., \& Egnell, M. (2021). Diet and physical activity during the coronavirus disease 2019 (COVID-19) lockdown(March-May 2020): results from the French NutriNet-Santé cohort study. Am.J.Clin. Nutr., 113(4), 924-938. https://doi.org/10.1093/ajcn/ nqaa336

Díaz, J. R., Fernández, C. A., Cabo, A. P., Pino, L. P., \& Gómez, C. A. (2019). Motivaciones y hábitos de actividad física en alumnos universitarios. Retos(36), 446-453.

Fayyad, F., \& Dopsaj, M. (2021). Level of Physical Activity at Lebanese Universities' Students of Both Genders: A Comparative Study. IJKSS, 9(3), 8-17. https://doi.org/ 10.7575/aiac.ijkss.v.9n.3p8

Franceschini, C., Musetti, A., Zenesini, C., Palagini, L., Scarpelli, S., Quattropani, M. C., Lenzo, V., Freda, M. F., Lemmo, D., \& Vegni, E. (2020). Poor sleep quality and its consequences on mental health during the COVID-19 lockdown in Italy. Front. Psychol., 11, 3072. https://doi.org/10.3389/fpsyg.2020.574475

Gallè, F., Sabella, E. A., Ferracuti, S., De Giglio, O., Caggiano, G., Protano, C., Valeriani, F., Parisi, E. A., Valerio, G., \& Liguori, G. (2020). Sedentary behaviors and physical activity of Italian undergraduate students during lockdown at the time of CoViD» 19 pandemic. Int J Environ Health Res, 17(17), 6171. https:/ / doi.org/ 10.3390/ijerph17176171

Gallo, L. A., Gallo, T. F., Young, S. L., Moritz, K. M., \& Akison, L. K. (2020). The impact of isolation measures due to COVID-19 on energy intake and physical activity levels in Australian university students. Nutrients, 12(6), 1865. https://doi.org/10.3390/nu12061865

Ghrouz, A. K., Noohu, M. M., Manzar, M. D., Spence, D. W., BaHammam, A. S., \& Pandi-Perumal, S. (2019). Physical activity and sleep quality in relation to mental health among college students. Sleep Breath, 23(2), 627634. https://doi.org/10.1007/s11325-019-01780-z

Güner, H. R., Hasanoðlu, Ý., \& Akta ${ }^{\circ}$, F. (2020). COVID19: Prevention and control measures in community. Turk JMed Sci, 50(SI-1), 571-577. https: / /doi.org/10.3906/ sag-2004-146

Kruk, J. (2014). Health and economic costs of physical inactivity. Asian Pac. J. Cancer Prev., 15(18), 7499-7503. https: / /doi.org/10.7314/APJCP.2014.15.18.7499

Lakens, D. (2013). Calculating and reporting effect sizes to facilitate cumulative science: a practical primer for $\mathrm{t}$ tests and ANOVAs. Front. Psychol., 4, 863. https:// doi.org/10.3389/fpsyg.2013.00863

Leyton-Román, M., Cordón, C., Jiménez-Castuera, R., \& Hodgson, I. Q. J. R. I. d. M.y. C. d.l.A. F. y. d. D. (2021). Análisis de la actividad física y estilos de vida en estudiantes universitarios. Rev.int.med.cienc.act.fís.deporte, 21(81), pp.175-195. https://doi.org/10.15366/ rimcafd2021.81.012

Lomelí, H., Pérez-Olmos, I., Talero-Gutiérrez, C., Moreno, C., González-Reyes, R., Palacios, L., De la Peña, F., \& Muñoz-Delgado,J.(2008). Escalas y cuestionarios para evaluar el sueño: una revisión. Actas Esp Psiquiatr, 36(1).

López-Nuevo, C. E., Molina, J. S., \& Ureña, G. D. (2021). Adherence to healthy habits and academic performance in Vocational Education Students (Adherencia a hábitos saludables y Rendimiento Académico en estudiantes de Formación Profesional). Retos, 42, 118-125. 
Maia, B. R., \& Dias, P. C. (2020). Anxiety, depression and stress in university students: the impact of COVID-19. Estud. psicol., 37. https://doi.org/10.1590/1982$0275202037 \mathrm{e} 200067$

Mancini, A. D. (2020). Heterogeneous mental health consequences of COVID-19: Costs and benefits. Psychol Trauma, 12(S1), S15. https://doi.org/10.1037/ tra0000894

Marelli, S., Castelnuovo, A., Somma, A., Castronovo, V., Mombelli, S., Bottoni, D., Leitner, C., Fossati, A., \& Ferini-Strambi, L. (2021). Impact of COVID-19 lockdown on sleep quality in university students and administration staff. J Neurol, 268(1), 8-15. https:/ / doi.org/10.1007/s00415-020-10056-6

Martínez-Sanz, J. M., Gómez-Arenas, A., García-Jaén, M., Sospedra, I., Norte, A., \& Cortell-Tormo, J. M. (2018). Análisis de las acciones de promoción de la actividad física desarrolladas por las universidades públicas valencianas. Nutr Hosp, 35(6), 1401-1415. https://doi.org/ 10.20960/nh.1779

Mella, J. A., Nazar, G., Delgado, F. M. S., Navarrete, C. E. B., Angulo, Y. L., \& Cobo-Rendón, R. C. (2021). Variables sociocognitivas y su relación con la actividad física en estudiantes universitarios chilenos. Retos, 40, 76-85.

Mikkelsen, K., Stojanovska, L., Polenakovic, M., Bosevski, M., \& Apostolopoulos, V. (2017). Exercise and mental health. Maturitas, 106, 48-56. https: / /doi.org/10.1016/ j.maturitas.2017.09.003

Ministerio de Sanidad, S. S. e. I., el Ministerio de Educación, Cultura y Deporte. (2008). RED ESPANNOLA DE UNIVERSIDADES SALUDABLES (REUS). Recuperado octubre, 10, 2021, de https://www.mscbs.gob.es/profesionales/saludPublica/prevPromocion/promocion/ UniversidadesSaludables/REUS.htm

Monge, S., Zamalloa, P. L., Moros, M. J. S., Olaso, O. P., San Miguel, L. G., Varela, C., Ariza, S. R., Torres, M. C. V., Lucerón, M. d. C. O., \& Yuste, P. G. (2021). Lifting COVID-19 mitigation measures in Spain (May-June 2020). Enferm Infecc Microbiol Clin. https://doi.org/ 10.1016/j.eimc.2021.05.011

Monteiro, L. Z., Varela, A. R., Lira, B. A., Souza, P. d., Gomes, D. J. d. O., Contiero, L. C., \& Bonardi, J. M.T. (2019). Behavioral health risk profiles of physical education undergraduates. Mot.Rev.de Educ.Fis., 25.https:/ /doi.org/10.1590/s1980-6574201900040146

Nicola, M., Alsafi, Z., Sohrabi, C., Kerwan, A., Al-Jabir, A., Iosifidis, C., Agha, M., \& Agha, R. (2020). The socioeconomic implications of the coronavirus pandemic (COVID-19): A review. International journal of surgery, 78, 185.https://doi.org/10.1016/j.ijsu.2020.04.018
Olmedilla, A., Ortega, E., \& Candel, N. (2010). Ansiedad, depresión y práctica de ejercicio físico en estudiantes universitarias. Apunts. Medicina de l'Esport, 45(167), 175180. https://doi.org/10.1016/j.apunts.2010.03.001

Organización de las Naciones Unidas, O. (2015). Agenda 2030 para el Desarrollo Sostenible. https: / /www.un.org/ sustainabledevelopment/es/2015/09/la-asamblea-general-adopta-la-agenda-2030-para-el-desarrollo-sostenible/

Organización Mundial de la Salud, O., \& Organización Paramericana de la Salud, O. (2020). La semana del Bienestar. Recuperado octubre, 10, 2021, de https: // www.paho.org/es/campa\%C3\%B1as/semana-bienestar-2020

Padrón, I., Fraga, I., Vieitez, L., Montes, C., \& Romero, E. (2021). A study on the psychological wound of COVID19 in university students. Front. Psychol., 12, 9. https:/ / doi.org/10.3389/fpsyg.2021.589927

Sachs, J. D. (2012). From millennium development goals to sustainable development goals. Lancet, 379(9832), 22062211. https://doi.org/10.1016/S0140-6736(12)606850

Santamaría, M. C., Cámara, M. C. P., \& Eguizábal, J. A. J. (2019). Diferencias en los motivos hacia la actividad física de los universitarios según nivel de actividad y su relación con la satisfacción del servicio deportivo universitario. Retos(35), 191-195.

Sun, J., Cheng,W., Fan, Z., \& Zhang, X. (2020). Influence of high-intensity intermittent training on glycolipid metabolism in obese male college students. Ann. Palliat. Med., 9, 2013-2019. https: / /doi.org/10.21037/apm-201105

Toloza, S. M., \& Gómez-Conesa, A. (2007). El Cuestionario Internacional de Actividad Física. Un instrumento adecuado en el seguimiento de la actividad física poblacional. Rev. iberoam. fisioter. kinesiol., 10(1), 48-52. https: / /doi.org/10.1016/S1138-6045(07)73665-1

Torales, J., Almirón, M., González, I., Gómez, N., Ortiz, R., \& Ibarra, V. (2018). Ejercicio físico como tratamiento adyuvante de los trastornos mentales. Una revisión narrativa. An. Fac. Cienc. Méd., 51(3), 27-32. https:// doi.org/10.18004/anales/2018.051(03)27-032

Vindegaard, N., \& Benros, M. E. (2020). COVID-19 pandemic and mental health consequences: Systematic review of the current evidence. Brain Behav., 89, 531542.https://doi.org/10.1016/j.bbi.2020.05.048 